\title{
A Subclass of Harmonic Univalent Functions Associated with $q$-Analogue of Dziok-Srivastava Operator
}

\author{
Huda Aldweby and Maslina Darus \\ School of Mathematical Sciences, Faculty of Science and Technology, Universiti Kebangsaan Malaysia, \\ 43600 Bangi, Selangor, Malaysia \\ Correspondence should be addressed to Maslina Darus; maslina@ukm.my
}

Received 26 June 2013; Accepted 1 August 2013

Academic Editors: G. Ólafsson and D.-X. Zhou

Copyright (C) 2013 H. Aldweby and M. Darus. This is an open access article distributed under the Creative Commons Attribution License, which permits unrestricted use, distribution, and reproduction in any medium, provided the original work is properly cited.

We study a class of complex-valued harmonic univalent functions using a generalized operator involving basic hypergeometric function. Precisely, we give a necessary and sufficient coefficient condition for functions in this class. Distortion bounds, extreme points, and neighborhood of such functions are also considered.

\section{Introduction}

Let $\mathbb{U}=\{z \in \mathbb{C}:|z|<1\}$ be the open unit disc, and let $S_{H}$ denote the class of functions which are complex valued, harmonic, univalent, and sense preserving in $\mathbb{U}$ normalized by $f(0)=f_{z}(0)-1=0$. Each $f \in S_{H}$ can be expressed as $f=h+\bar{g}$, where $h$ and $g$ are analytic in $\mathbb{U}$. We call $h$ the analytic part and $g$ the coanalytic part of $f$. A necessary and sufficient condition for $f$ to be locally univalent and sense preserving in $\mathbb{U}$ is that $\left|h^{\prime}(z)\right|>\left|g^{\prime}(z)\right|$ in $\mathbb{U}$ (see [1]). In [2], there is a more comprehensive study on harmonic univalent functions. Thus, for $f=h+\bar{g} \in S_{H}$, we may write

$$
h(z)=z+\sum_{k=2}^{\infty} a_{k} z^{k}, \quad g(z)=\sum_{k=1}^{\infty} b_{k} z^{k} \quad\left(0 \leq b_{1}<1\right) .
$$

Note that $S_{H}$ reduces to $S$, the class of normalized analytic univalent functions, if the coanalytic part of $f=h+\bar{g}$ is identically zero.

The study of basic hypergeometric series (also called $q$ hypergeometric series) essentially started in 1748 when Euler considered the infinite product $(q ; q)_{\infty}^{-1}=\prod_{k=0}^{\infty}\left(1-q^{k+1}\right)^{-1}$. In the literature, we were told that the development of these functions was much slower until, in 1878, Heine converted a simple observation that $\lim _{q \rightarrow 1}\left[\left(1-q^{a}\right) /(1-q)\right]=a$ which returns the theory of ${ }_{2} \phi_{1}$ basic hypergeometric series to the famous theory of Gauss's ${ }_{2} F_{1}$ hypergeometric series. The importance of basic hypergeometric functions is due to their application in deriving $q$-analogue of well-known functions, such as $q$-analogues of the exponential, gamma, and beta functions. In this paper, we define a class of starlike harmonic functions using basic hypergeometric functions and investigate its properties like coefficient condition, distortion theorem, and extreme points.

For complex parameters $a_{i}, b_{j}, q(i=1, \ldots, r, j=1, \ldots, s$, $\left.b_{j} \in \mathbb{C} \backslash\{0,-1,-2, \ldots\},|q|<1\right)$, we define the basic hypergeometric function $\Phi_{r} \Phi_{s}\left(a_{1}, \ldots, a_{r} ; b_{1}, \ldots, b_{s}, q, z\right)$ by

$$
\begin{aligned}
{ }_{r} \Phi_{s} & \left(a_{1} \ldots, a_{r} ; b_{1}, \ldots, b_{s}, q, z\right) \\
& =\sum_{k=0}^{\infty} \frac{\left(a_{1}, q\right)_{k} \cdots\left(a_{r}, q\right)_{k}}{(q, q)_{k}\left(b_{1}, q\right)_{k} \cdots\left(b_{s}, q\right)_{k}} z^{k},
\end{aligned}
$$

$\left(r=s+1 ; r, s \in \mathbb{N}_{0}=\mathbb{N} \cup\{0\} ; z \in \mathbb{U}\right)$ where $\mathbb{N}$ denote the set of positive integers and $(a, q)_{k}$ is the $q$-shifted factorial defined by

$$
\begin{aligned}
& (a, q)_{k} \\
& \quad= \begin{cases}1, & k=0 ; \\
(1-a)(1-a q)\left(1-a q^{2}\right) \cdots\left(1-a q^{k-1}\right), & k \in \mathbb{N} .\end{cases}
\end{aligned}
$$


We note that

$$
\begin{aligned}
\lim _{q \rightarrow 1^{-}} & {\left[{ }_{r} \Phi_{s}\left(q^{a_{1}}, \ldots, q^{a_{r}} ; q^{b_{1}}, \ldots, q^{b_{s}}, q,(q-1)^{1+s-r} z\right)\right] } \\
& ={ }_{r} F_{s}\left(a_{1}, \ldots, a_{r} ; b_{1}, \ldots, b_{s}, z\right),
\end{aligned}
$$

where ${ }_{r} F_{s}\left(a_{1}, \ldots, a_{r} ; b_{1}, \ldots, b_{s}, z\right)$ is the well-known generalized hypergeometric function. By the ratio test, one observes that for $|q|<1$ and $r=s+1$ the series defined in (2) converges absolutely in $\mathbb{U}$ so that it represented an analytic function in $\mathbb{U}$. For more mathematical background of basic hypergeometric functions, one may refer to $[3,4]$.

The $q$-derivative of a function $h(x)$ is defined by

$$
D_{q}(h(x))=\frac{h(q x)-h(x)}{(q-1) x}, \quad q \neq 1, x \neq 0 .
$$

For a function $h(z)=z^{k}$, we can observe that

$$
D_{q}(h(z))=D_{q}\left(z^{k}\right)=\frac{1-q^{k}}{1-q} z^{k-1}=[k]_{q} z^{k-1} .
$$

Then $\lim _{q \rightarrow 1} D_{q}(h(z))=\lim _{q \rightarrow 1}[k]_{q} z^{k-1}=k z^{k-1}=h^{\prime}(z)$, where $h^{\prime}(z)$ is the ordinary derivative. For more properties of $D_{q}$, see $[4,5]$.

Corresponding to the function ${ }_{r} \Phi_{s}\left(a_{1}, \ldots, a_{r} ; b_{1}, \ldots, b_{s}\right.$, $q, z)$, consider

$$
\begin{aligned}
{ }_{r} \mathscr{G}_{s} & \left(a_{1}, \ldots, a_{r} ; b_{1}, \ldots, b_{s}, q, z\right) \\
& =z_{r} \Phi_{s}\left(a_{1}, \ldots, a_{r} ; b_{1}, \ldots, b_{s}, q, z\right) \\
& =z+\sum_{k=2}^{\infty} \frac{\left(a_{1}, q\right)_{k-1} \cdots\left(a_{r}, q\right)_{k-1}}{(q, q)_{k-1}\left(b_{1}, q\right)_{k-1} \cdots\left(b_{s}, q\right)_{k-1}} z^{k}
\end{aligned}
$$

The authors [6] defined the linear operator $H_{s}^{r}\left(a_{1}, \ldots, a_{r}\right.$; $\left.b_{1}, \ldots, b_{s} ; q\right) f: \mathscr{A} \rightarrow \mathscr{A}$ by

$$
\begin{aligned}
H_{s}^{r} & \left(a_{1}, \ldots, a_{r} ; b_{1}, \ldots, b_{s} ; q\right) f(z) \\
& ={ }_{r} \mathscr{G}_{s}\left(a_{1}, \ldots, a_{r} ; b_{1}, \ldots, b_{s}, q, z\right) * f(z) \\
& =z+\sum_{k=2}^{\infty} \Gamma\left(a_{1}, q, k\right) a_{k} z^{k},
\end{aligned}
$$

where $(*)$ stands for convolution and

$$
\Gamma\left(a_{1}, q, k\right)=\frac{\left(a_{1}, q\right)_{k-1} \cdots\left(a_{r}, q\right)_{k-1}}{(q, q)_{k-1}\left(b_{1}, q\right)_{k-1} \cdots\left(b_{s}, q\right)_{k-1}} .
$$

To make the notation simple, we write

$$
H_{s}^{r}\left[a_{1}, q\right] f(z)=H_{s}^{r}\left(a_{1}, \ldots, a_{r} ; b_{1}, \ldots, b_{s} ; q\right) f(z) \text {. }
$$

We define the operator (8) of harmonic function $f=h+\bar{g}$ given by (1) as

$$
H_{s}^{r}\left[a_{1}, q\right] f(z)=H_{s}^{r}\left[a_{1}, q\right] h(z)+\overline{H_{s}^{r}\left[a_{1}, q\right] g(z)} .
$$

Definition 1. For $0 \leq \delta<1$, let $S_{H}^{*}\left(a_{1}, \delta, q\right)$ denote the subfamily of starlike harmonic functions $f \in S_{H}^{*}$ of the form (1) such that

$$
\frac{\partial}{\partial \theta}\left(\arg H_{s}^{r}\left[a_{1}, q\right] f\right) \geq \delta, \quad|z|=r<1 .
$$

Following [7], a function $f$ is said to be in the class $V_{\bar{H}}\left(a_{1}, \delta, q\right)=S_{H}^{*}\left(a_{1}, \delta, q\right) \cap V_{H}$ if $f$ of the form (1) satisfies the condition that

$$
\arg \left(a_{k}\right)=\theta_{k} \quad \arg \left(b_{k}\right)=\vartheta_{k} \quad(k \geq n+1 ; n \in \mathbb{N})
$$

and if there exists a real number $\rho$ such that

$$
\begin{aligned}
\theta_{k}+(k-1) \phi \equiv \pi(\bmod 2 \pi), & \vartheta_{k}+(k-1) \phi \equiv 0, \\
& (k \geq n+1 ; n \in \mathbb{N}) .
\end{aligned}
$$

By specializing the parameters of $H_{s}^{r}\left[a_{1}, q\right] f$, we obtain different classes of starlike harmonic functions, for example,

(i) for $r=s+1, a_{2}=b_{1}, \ldots, a_{r}=b_{s}, S_{H}^{*}(q, q, \delta)=$ $S H(\delta)$ [8] is the class of sense-preserving harmonic univalent functions $f$ which are starlike of order $\delta$ in $\mathbb{U}$; that is, $\partial / \partial \theta\left(\arg f\left(r e^{i \theta}\right)\right) \geq \delta$;

(ii) for $r=s+1, a_{2}=b_{1}, \ldots, a_{r}=b_{s}$, and $a_{1}=$ $q^{n+1}, q \rightarrow 1, S_{H}^{*}\left(q^{n+1}, q, \delta\right)=R_{H}(n, \alpha)$ [9] is the class of starlike harmonic univalent functions with $(\partial / \partial \theta)\left(\arg D^{n} f(z)\right) \geq \delta$, where $D$ is the Ruscheweyh derivative (see [10]);

(iii) for $i=\{1, \ldots, r\}, j=\{1, \ldots, s\}, r=s+1, a_{i}=q^{\alpha_{i}}$, and $b_{j}=q^{\beta_{j}}, q \rightarrow 1, S_{H}^{*}\left(a_{1}, q, \delta\right)=S_{H}^{*}\left(\alpha_{1}, \delta\right)[11]$ is the class of starlike harmonic univalent functions with $(\partial / \partial \theta)\left(\arg H_{s}^{r}\left[\alpha_{1}\right] f\right) \geq \delta$, where $H_{s}^{r}\left[\alpha_{1}\right]$ is the Dziok-Srivastava operator (see [12]).

\section{Main Results}

In our first theorem, we introduce a sufficient coefficient bound for harmonic functions in $S_{H}^{*}\left(a_{1}, \delta, q\right)$.

Theorem 2. Let $f=h+\bar{g}$ be given by (1). If

$$
\begin{aligned}
& \sum_{k=2}^{\infty}\left(\frac{[k]_{q}-\delta}{1-\delta}\left|a_{k}\right|+\frac{[k]_{q}+\delta}{1-\delta}\left|b_{k}\right|\right) \Gamma\left(a_{1}, q, k\right) \\
& \quad \leq 1-\frac{1+\delta}{1-\delta}\left|b_{1}\right|
\end{aligned}
$$

where $a_{1}=1,0 \leq \delta<1$, and $\Gamma\left(a_{1}, q, k\right)$ is given by (9), then $f \in S_{H}^{*}\left(a_{1}, \delta, q\right)$. 
Proof. To prove that $f \in S_{H}^{*}\left(a_{1}, \delta, q\right)$, we only need to show that if (15) holds, then the required condition (12) is satisfied. For (12), we can write

$$
\begin{aligned}
\frac{\partial}{\partial \theta}( & \left.\arg H_{s}^{r}\left[a_{1}, q\right] f(z)\right) \\
=\Re & \left\{\frac{z D_{q}\left(H_{s}^{r}\left[a_{1}, q\right] h(z)\right)}{H_{s}^{r}\left[a_{1}, q\right] h(z)+\overline{H_{s}^{r}\left[a_{1}, q\right] g(z)}}\right. \\
& \left.\quad-\frac{\overline{z D_{q}\left(H_{s}^{r}\left[a_{1}, q\right] g(z)\right)}}{H_{s}^{r}\left[a_{1}, q\right] h(z)+\overline{H_{s}^{r}\left[a_{1}, q\right] g(z)}}\right\} \\
= & \Re \frac{A(z)}{B(z)} .
\end{aligned}
$$

Using the fact that $\mathfrak{R}(w) \geq \delta$ if and only if $|1-\delta+w| \geq$ $|1+\delta-w|$, it suffices to show that

$$
|A(z)+(1-\delta) B(z)|-|A(z)-(1+\delta) B(z)| \geq 0 .
$$

Substituting for $A(z)$ and $B(z)$ in (15) yields

$$
\begin{aligned}
& |A(z)+(1-\delta) B(z)|-|A(z)-(1+\delta) B(z)| \\
& \geq(2-\delta)|z|-\sum_{k=2}^{\infty}\left([k]_{q}+1-\delta\right) \Gamma\left(a_{1}, q, k\right)\left|a_{k}\right||z|^{k} \\
& \quad-\sum_{k=1}^{\infty}\left([k]_{q}-1+\delta\right) \Gamma\left(a_{1}, q, k\right)\left|b_{k}\right||z|^{k} \\
& \quad-\delta|z|-\sum_{k=2}^{\infty}\left([k]_{q}-1-\delta\right) \Gamma\left(a_{1}, q, k\right)\left|a_{k}\right||z|^{k} \\
& \quad-\sum_{k=1}^{\infty}\left([k]_{q}+1+\delta\right) \Gamma\left(a_{1}, q, k\right)\left|b_{k}\right||z|^{k} \\
& \geq 2(1-\delta)|z|\left\{1-\sum_{k=2}^{\infty} \frac{[k]_{q}-\delta}{1-\delta} \Gamma\left(a_{1}, q, k\right)\left|a_{k}\right|\right.
\end{aligned}
$$

$$
\left.-\sum_{k=1}^{\infty} \frac{[k]_{q}+\delta}{1-\delta} \Gamma\left(a_{1}, q, k\right)\left|b_{k}\right|\right\}
$$

$$
\begin{aligned}
=2(1-\delta)|z| & \left\{1-\frac{1+\delta}{1-\delta}\left|b_{1}\right|\right. \\
& -\left[\sum_{k=2}^{\infty}\left(\frac{[k]_{q}-\delta}{1-\delta}\left|a_{k}\right|+\frac{[k]_{q}+\delta}{1-\delta}\left|b_{k}\right|\right)\right] \\
& \left.\times \Gamma\left(a_{1}, q, k\right)\right\} .
\end{aligned}
$$

The last expression is nonnegative by (15), and so, $f \in$ $S_{H}^{*}\left(a_{1}, \delta, q\right)$.

Now, we obtain the necessary and sufficient conditions for $f=h+\bar{g}$ given by (14).

Theorem 3. Let $f=h+\bar{g}$ be given by (11). Then, $f \in$ $V_{\bar{H}}\left(a_{1}, \delta, q\right)$ if and only if

$$
\begin{aligned}
& \sum_{k=2}^{\infty}\left(\frac{[k]_{q}-\delta}{1-\delta}\left|a_{k}\right|+\frac{[k]_{q}+\delta}{1-\delta}\left|b_{k}\right|\right) \Gamma\left(a_{1}, q, k\right) \\
& \quad \leq 1-\frac{1+\delta}{1-\delta}\left|b_{1}\right|
\end{aligned}
$$

where $a_{1}=1,0 \leq \delta<1$, and $\Gamma\left(a_{1}, q, k\right)$ is given by (9).

Proof. Since $V_{\bar{H}}\left(a_{1}, \delta, q\right) \subset S_{H}^{*}\left(a_{1}, \delta, q\right)$, we only to prove the only if part of the theorem. So that for functions $f \in V_{\bar{H}}\left(a_{1}, \delta, q\right)$, we notice that the condition $(\partial / \partial \theta)\left(\arg H_{s}^{r}\left[a_{1}, q\right] f(z)\right) \geq \delta$ is equivalent to

$$
\begin{aligned}
& \frac{\partial}{\partial \theta}\left(\arg H_{s}^{r}\left[a_{1}, q\right] f(z)\right)-\delta \\
&=\Re\left\{\frac{z D_{q}\left(H_{s}^{r}\left[a_{1}, q\right] h(z)\right)}{H_{s}^{r}\left[a_{1}, q\right] h(z)+\overline{H_{s}^{r}\left[a_{1}, q\right] g(z)}}\right. \\
&\left.\quad-\frac{\overline{z D_{q}\left(H_{s}^{r}\left[a_{1}, q\right] g(z)\right)}}{H_{s}^{r}\left[a_{1}, q\right] h(z)+\overline{H_{s}^{r}\left[a_{1}, q\right] g(z)}}-\delta\right\} \geq 0 .
\end{aligned}
$$

That is,

$$
\mathfrak{R}\left[\frac{(1-\delta) z+\sum_{k=2}^{\infty}\left([k]_{q}-\delta\right) \Gamma\left(a_{1}, q, k\right)\left|a_{k}\right| z^{k}-\sum_{k=1}^{\infty}\left([k]_{q}+\delta\right) \overline{\Gamma\left(a_{1}, q, k\right)}\left|b_{k}\right| \bar{z}^{k}}{z+\sum_{k=2}^{\infty} \Gamma\left(a_{1}, q, k\right)\left|a_{k}\right| z^{k}+\sum_{k=1}^{\infty} \overline{\Gamma\left(a_{1}, q, k\right)}\left|b_{k}\right| \bar{z}^{k}}\right] \geq 0 .
$$


The previous condition must hold for all values of $z$ in $\mathbb{U}$. Upon choosing $\phi$ according to (14), we must have

$$
\begin{aligned}
& \frac{(1-\delta)-(1+\delta)\left|b_{1}\right|}{1+\left|b_{1}\right|+\sum_{k=2}^{\infty}\left(\left|a_{k}\right|+\left|b_{k}\right|\right) \Gamma\left(a_{1}, q, k\right) r^{k-1}} \\
& \quad-\frac{\sum_{k=2}^{\infty}\left(\left([k]_{q}-\delta\right)\left|a_{k}\right|+\left([k]_{q}+\delta\right)\left|b_{k}\right|\right) \Gamma\left(a_{1}, q, k\right) r^{k-1}}{1+\left|b_{1}\right|+\sum_{k=2}^{\infty}\left(\left|a_{k}\right|+\left|b_{k}\right|\right) \Gamma\left(a_{1}, q, k\right) r^{k-1}} \\
& \quad \geq 0
\end{aligned}
$$

If condition (19) does not hold, then the numerator in (22) is negative for $r$ sufficiently close to 1 . Hence, there exist $z_{0}=r_{0}$ in $(0,1)$ for which the quotient of $(22)$ is negative. This contradicts the fact that $f \in V_{\bar{H}}\left(a_{1}, \delta, q\right)$, and this completes the proof.

The following theorem gives the distortion bounds for functions in $V_{\bar{H}}\left(a_{1}, \delta, q\right)$ which yield a covering result for this class.

Theorem 4. If $f \in V_{\bar{H}}\left(a_{1}, \delta, q\right)$, then

$$
\begin{aligned}
|f(z)| \leq & \left(1+\left|b_{1}\right|\right) r \\
& +\frac{1}{\Gamma\left(a_{1}, q, 2\right)}\left(\frac{1-\delta}{(q+1)-\delta}-\frac{1+\delta}{(q+1)-\delta}\right) r^{2} \\
& |z|=r<1, \\
|f(z)| \geq\left(1+\left|b_{1}\right|\right) r & -\frac{1}{\Gamma\left(a_{1}, q, 2\right)}\left(\frac{1-\delta}{(q+1)-\delta}-\frac{1+\delta}{(q+1)-\delta}\right) r^{2} \\
& \quad|z|=r<1,
\end{aligned}
$$

where

$$
\begin{gathered}
\Gamma\left(a_{1}, q, 2\right)=\frac{\left(1-a_{1}\right) \cdots\left(1-a_{r}\right)}{(1-q)\left(1-b_{1}\right) \cdots\left(1-b_{s}\right)}, \\
{[2]_{q}=(q+1) .}
\end{gathered}
$$

Proof. We will only prove the right hand inequality. The proof for the left hand inequality is similar. obtain

let $f \in V_{\bar{H}}\left(a_{1}, \delta, q\right)$. Taking the absolute value of $f$, we

$$
\begin{aligned}
|f(z)| & \leq\left(1+\left|b_{1}\right|\right) r+\sum_{k=2}^{\infty}\left(\left|a_{k}\right|+\left|b_{k}\right|\right) r^{k} \\
& \leq\left(1+\left|b_{1}\right|\right) r+\sum_{k=2}^{\infty}\left(\left|a_{k}\right|+\left|b_{k}\right|\right) r^{2} .
\end{aligned}
$$

That is,

$$
\begin{aligned}
|f(z)| \leq & \left(1+\left|b_{1}\right|\right) r+\frac{1-\delta}{\Gamma\left(a_{1}, q, 2\right)\left([2]_{q}-\delta\right)} \\
& \times \sum_{k=2}^{\infty}\left(\frac{[2]_{q}-\delta}{1-\delta}\left|a_{k}\right|+\frac{[2]_{q}-\delta}{1-\delta}\left|b_{k}\right|\right) \\
& \times \Gamma\left(a_{1}, q, 2\right) r^{2} \\
\leq & \left(1+\left|b_{1}\right|\right) r+\frac{1-\delta}{\Gamma\left(a_{1}, q, 2\right)((q+1)-\delta)} \\
& \times\left[1-\frac{1+\delta}{1-\delta}\left|b_{1}\right|\right] r^{2} \\
& =\left(1+\left|b_{1}\right|\right) r+\frac{1}{\Gamma\left(a_{1}, q, 2\right)} \\
& \times\left[\frac{1-\delta}{(q+1)-\delta}-\frac{1+\delta}{(q+1)-\delta}\left|b_{1}\right|\right) r^{2} .
\end{aligned}
$$

Corollary 5. Let $f$ be of the form (1) so that $f \in V_{\bar{H}}\left(a_{1}, \delta, q\right)$. Then,

$$
\begin{aligned}
& \left\{w:|w|<\frac{2 \Gamma\left(a_{1}, q, 2\right)-1-\left(\Gamma\left(a_{1}, q, 2\right)-1\right) \delta}{((q+1)-\delta) \Gamma\left(a_{1}, q, 2\right)}\right. \\
& \left.\quad-\frac{2 \Gamma\left(a_{1}, q, 2\right)-1-\left(\Gamma\left(a_{1}, q, 2\right)-1\right) \delta}{((q+1)+\delta) \Gamma\left(a_{1}, q, 2\right)}\left|b_{1}\right|\right\} \subset f(\mathbb{U}) .
\end{aligned}
$$

Next, one determines the extreme points of closed convex hull of $V_{\bar{H}}\left(a_{1}, \delta, q\right)$ denoted by $\operatorname{clco} V_{\bar{H}}\left(a_{1}, \delta, q\right)$.

Theorem 6. Set

$$
\begin{aligned}
& \lambda_{k}=\frac{1-\delta}{\left([k]_{q}-\delta\right) \Gamma\left(a_{1}, q, k\right)}, \\
& \mu_{k}=\frac{1-\delta}{\left([k]_{q}+\delta\right) \Gamma\left(a_{1}, q, k\right)} .
\end{aligned}
$$

For $b_{1}$ fixed, the extreme points for clco $V_{\bar{H}}\left(a_{1}, \delta, q\right)$ are

$$
\left\{z+\lambda_{k} x z^{k}+\overline{b_{1} z}\right\} \cup\left\{z+\overline{b_{1} z+\mu_{k} x z^{k}}\right\}
$$

where $k \geq 2$ and $|x|=1-\left|b_{1}\right|$.

Proof. Any function $f \in \operatorname{clco} V_{\bar{H}}\left(a_{1}, \delta, q\right)$ may be expressed as

$$
f(z)=z+\sum_{k=2}^{\infty}\left|a_{k}\right| e^{i \alpha_{k}} z^{k}+\overline{b_{1} z}+\overline{\sum_{k=2}^{\infty}\left|b_{k}\right| e^{i \beta_{k}} z^{k}}
$$


where the coefficients satisfy the inequality (15). Set $h_{1}(z)=$ $z, g_{1}(z)=b_{1} z, h_{k}(z)=z+\lambda_{k} e^{i \alpha_{k}} z^{k}$, and $g_{k}(z)=b_{1}(z)+$ $e^{i \beta_{k}} z^{k}$, for $k=2,3, \ldots$ Writing $X_{k}=\left|a_{k}\right| / \lambda_{k}, Y_{k}=\left|b_{k}\right| / \mu_{k}$, $k=2,3, \ldots$ and $X_{1}=1-\sum_{k=2}^{\infty} X_{k} ; Y_{1}=1-\sum_{k=2}^{\infty} Y_{k}$, we have

$$
f(z)=\sum_{k=1}^{\infty}\left(X_{k} h_{k}(z)+Y_{k} g_{k}(z)\right) .
$$

In particular, set

$$
\begin{array}{r}
f_{1}(z)=z+\overline{b_{1} z}, \quad f_{k}(z)=z+\lambda_{k} x z^{k}+\overline{b_{1} z}+\overline{\mu_{k} y z^{k}} \\
\left(k \geq 2,|x|+|y|=1-\left|b_{1}\right|\right) .
\end{array}
$$

Therefore, the extreme points of clco $V_{\bar{H}}\left(a_{1}, \delta, q\right)$ are contained in $\left\{f_{k}(z)\right\}$. To see that $f_{1}$ is not an extreme point, note that $f_{1}$ may be written as a convex linear combination of functions in $\operatorname{clco} V_{\bar{H}}\left(a_{1}, \delta, q\right)$ as follows:

$$
\begin{aligned}
f_{1}(z)= & \frac{1}{2}\left\{f_{1}(z)+\lambda_{2}\left(1-\left|b_{1}\right|\right) z^{2}\right\} \\
& +\frac{1}{2}\left\{f_{1}(z)-\lambda_{2}\left(1-\left|b_{1}\right|\right) z^{2}\right\} .
\end{aligned}
$$

If both $|x| \neq 0$ and $|y| \neq 0$, we will show that it can also be expressed as a convex linear combination of functions in clco $V_{\bar{H}}\left(a_{1}, \delta, q\right)$. Without loss of generality, assume that $|x| \geq$ $|y|$. Choose $\epsilon>0$ small enough so that $\epsilon<|x| /|y|$. Set $A=1+\epsilon$ and $B=1-|\epsilon x / y|$. We then see that both

$$
\begin{gathered}
t_{1}(z)=z+\lambda_{k} A x z^{k}+\overline{b_{1} z}+\overline{\mu_{k} y B z^{k}} \\
t_{2}(z)=z+\lambda_{k}(2-A) x z^{k}+\overline{b_{1} z}+\overline{\mu_{k} y(2-B) z^{k}}
\end{gathered}
$$

are in $\operatorname{clco} V_{\bar{H}}\left(a_{1}, \delta, q\right)$ and note that

$$
f_{n}(z)=\frac{1}{2}\left\{t_{1}(z)+t_{2}(z)\right\} .
$$

The extremal coefficient bound shows that the functions of the form (29) are extreme points for $\operatorname{clco} V_{\bar{H}}\left(a_{1}, \delta, q\right)$, and so the proof is complete.

Following the earlier works in $[2,13]$, we refer to the $\gamma$ neighborhood of the functions $f$ defined by (1) to be the set of functions $F$ for which

$$
\begin{aligned}
N_{\gamma}(f)=\{F & =z+\sum_{k=2}^{\infty} A_{k} z^{k}+\sum_{k=1}^{\infty} \overline{B_{k} z^{k}} \\
& \left.: \sum_{k=2}^{\infty} k\left(\left|a_{k}-A_{k}\right|+\left|b_{k}-B_{k}\right|\right)+\left|b_{1}-B_{1}\right| \leq \gamma\right\} .
\end{aligned}
$$

We define the $q$ - $\gamma$-neighborhood of a function $f \in S_{H}$ as follows:

$$
\begin{aligned}
& N_{\gamma}^{q}(f)=\left\{F=z+\sum_{k=2}^{\infty} A_{k} z^{k}+\sum_{k=1}^{\infty} \overline{B_{k} z^{k}}\right. \\
& : \sum_{k=2}^{\infty}[k]_{q}\left(\left|a_{k}-A_{k}\right|+\left|b_{k}-B_{k}\right|\right)
\end{aligned}
$$

$$
\left.+\left|b_{1}-B_{1}\right| \leq \gamma\right\} \text {. }
$$

In our case, let us define the generalized $q-\gamma$ neighborhood of $f$ to be the set

$$
\begin{aligned}
N_{\gamma}^{q}(f)=\left\{F: \sum_{k=2}^{\infty} \Gamma\left(a_{1}, q, k\right)\left[\left([k]_{q}-\delta\right)\left|a_{k}-A_{k}\right|\right.\right. \\
\left.+\left([k]_{q}+\delta\right)\left|b_{k}-B_{k}\right|\right] \\
\left.+(1+\delta)\left|b_{1}-B_{1}\right| \leq(1-\delta) \gamma\right\}
\end{aligned}
$$

Theorem 7. Let $f$ be given by (1). If $f$ satisfies the conditions

$$
\begin{aligned}
& \sum_{k=2}^{\infty}[k]_{q}\left([k]_{q}-\delta\right)\left|a_{k}\right| \Gamma\left(a_{1}, q, k\right) \\
& +\sum_{1=2}^{\infty}[k]_{q}\left([k]_{q}+\delta\right)\left|b_{k}\right| \Gamma\left(a_{1}, q, k\right) \leq 1-\delta, \\
& \qquad 0 \leq \delta<1, \\
& \gamma \leq \frac{1-\delta}{(q+1)-\delta}\left(1-\frac{1+\delta}{1-\delta}\left|b_{1}\right|\right),
\end{aligned}
$$

then $N_{\gamma}^{q}(f) \subset S_{H}^{*}\left(a_{1}, \delta, q\right)$.

Proof. Let $f$ satisfy (39) and let

$$
F(z)=z+\overline{B_{1} z}+\sum_{k=2}^{\infty}\left(A_{k} z^{k}+\overline{B_{k} z^{k}}\right)
$$

belong to $N_{\gamma}^{q}(f)$. We have

$$
\begin{gathered}
(1+\delta)\left|B_{1}\right|+\sum_{k=2}^{\infty} \Gamma\left(a_{1}, q, k\right)\left[\left([k]_{q}-\delta\right)\left|A_{k}\right|\right. \\
\left.+\left([k]_{q}+\delta\right)\left|B_{k}\right|\right] \\
\leq(1+\delta)\left|B_{1}-b_{1}\right|+(1+\delta)\left|b_{1}\right|
\end{gathered}
$$




$$
\begin{aligned}
& +\sum_{k=2}^{\infty} \Gamma\left(a_{1}, q, k\right)\left[\left([k]_{q}-\delta\right)\left|A_{k}-a_{k}\right|\right. \\
& \left.+\left([k]_{q}+\delta\right)\left|B_{k}-b_{k}\right|\right] \\
& +\sum_{k=2}^{\infty} \Gamma\left(a_{1}, q, k\right)\left[\left([k]_{q}-\delta\right)\left|a_{k}\right|+\left([k]_{q}+\delta\right)\left|b_{k}\right|\right] \\
& \leq(1-\delta) \gamma+(1+\delta)\left|b_{1}\right| \\
& +\frac{1}{[2]_{q}-\delta} \sum_{k=2}^{\infty} \Gamma\left(a_{1}, q, k\right)\left[[k]_{q}\left([k]_{q}-\delta\right)\left|a_{k}\right|\right. \\
& \leq(1-\delta) \gamma+(1+\delta)\left|b_{1}\right|+\frac{1}{(q+1)-\delta} \\
& \quad \times\left[(1-\delta)-(1+\delta)\left|b_{1}\right|\right] \leq 1-\delta .
\end{aligned}
$$

Hence,

$$
\begin{gathered}
\gamma \leq \frac{1-\delta}{(q+1)-\delta}\left(1-\frac{1+\delta}{1-\delta}\left|b_{1}\right|\right), \\
F \in S_{H}^{*}\left(a_{1}, \delta, q\right) .
\end{gathered}
$$

\section{Acknowledgment}

The work presented here was partially supported by LRGS/ TD/2011/UKM/ICT/03/02 and UKM-DLP-2011-050.

\section{References}

[1] J. Clunie and T. Sheil-Small, "Harmonic univalent functions," Annales Academiae Scientiarum Fennicae A, vol. 9, pp. 3-25, 1984.

[2] Y. Avc1 and E. Złotkiewicz, "On harmonic univalent mappings," Annales Universitatis Mariae Curie-Skłodowska A, vol. 44, pp. 1-7, 1990.

[3] G. Gasper and M. Rahman, Basic Hypergeometric Series, vol. 35 of Encyclopedia of Mathematics and Its Applications, Cambridge University Press, Cambridge, UK, 1990.

[4] H. Exton, q-hypergeometric functions and applications, Ellis Horwood Series: Mathematics and its Applications, Ellis Horwood, Chichester, UK, 1983.

[5] H. A. Ghany, " $q$-derivative of basic hypergeometric series with respect to parameters," International Journal of Mathematical Analysis, vol. 3, no. 33-36, pp. 1617-1632, 2009.

[6] A. Mohammed and M. Darus, "A generalized operator involving the q-hypergeometric function," Matematicki Vesnik, vol. 65, no. 4, pp. 454-465, 2013.

[7] J. M. Jahangiri and H. Silverman, "Harmonic univalent functions with varying arguments," International Journal of Applied Mathematics, vol. 8, no. 3, pp. 267-275, 2002.

[8] J. M. Jahangiri, "Harmonic functions starlike in the unit disk," Journal of Mathematical Analysis and Applications, vol. 235, no. 2, pp. 470-477, 1999.
[9] G. Murugusundaramoorthy, "A class of Ruscheweyh-type harmonic univalent functions with varying arguments," Southwest Journal of Pure and Applied Mathematics, no. 2, pp. 90-95, 2003.

[10] S. Ruscheweyh, "New criteria for univalent functions," Proceedings of the American Mathematical Society, vol. 49, pp. 109-115, 1975.

[11] H. A. Al-Kharsani and R. A. Al-Khal, "Univalent harmonic functions," Journal of Inequalities in Pure and Applied Mathematics, vol. 8, no. 2, article 59, p. 8, 2007.

[12] J. Dziok and H. M. Srivastava, "Classes of analytic functions associated with the generalized hypergeometric function," Applied Mathematics and Computation, vol. 103, no. 1, pp. 1-13, 1999.

[13] S. Ruscheweyh, "Neighborhoods of univalent functions," Proceedings of the American Mathematical Society, vol. 81, no. 4, pp. 521-527, 1981. 


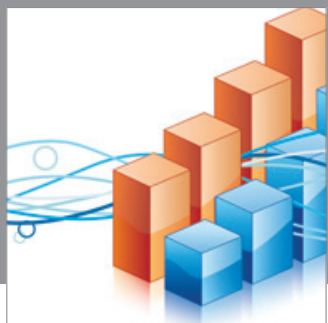

Advances in

Operations Research

mansans

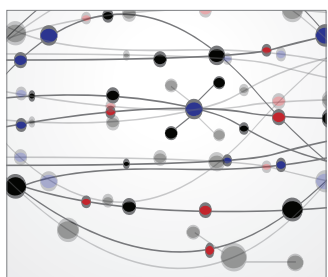

The Scientific World Journal
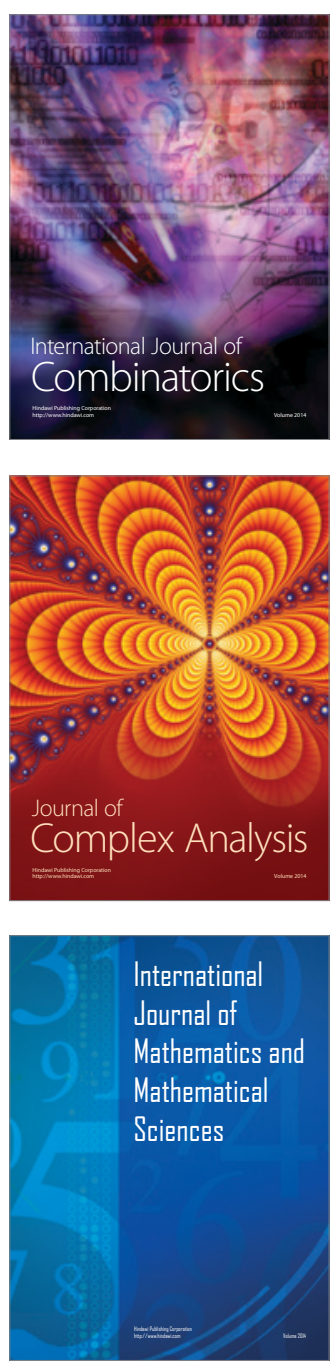
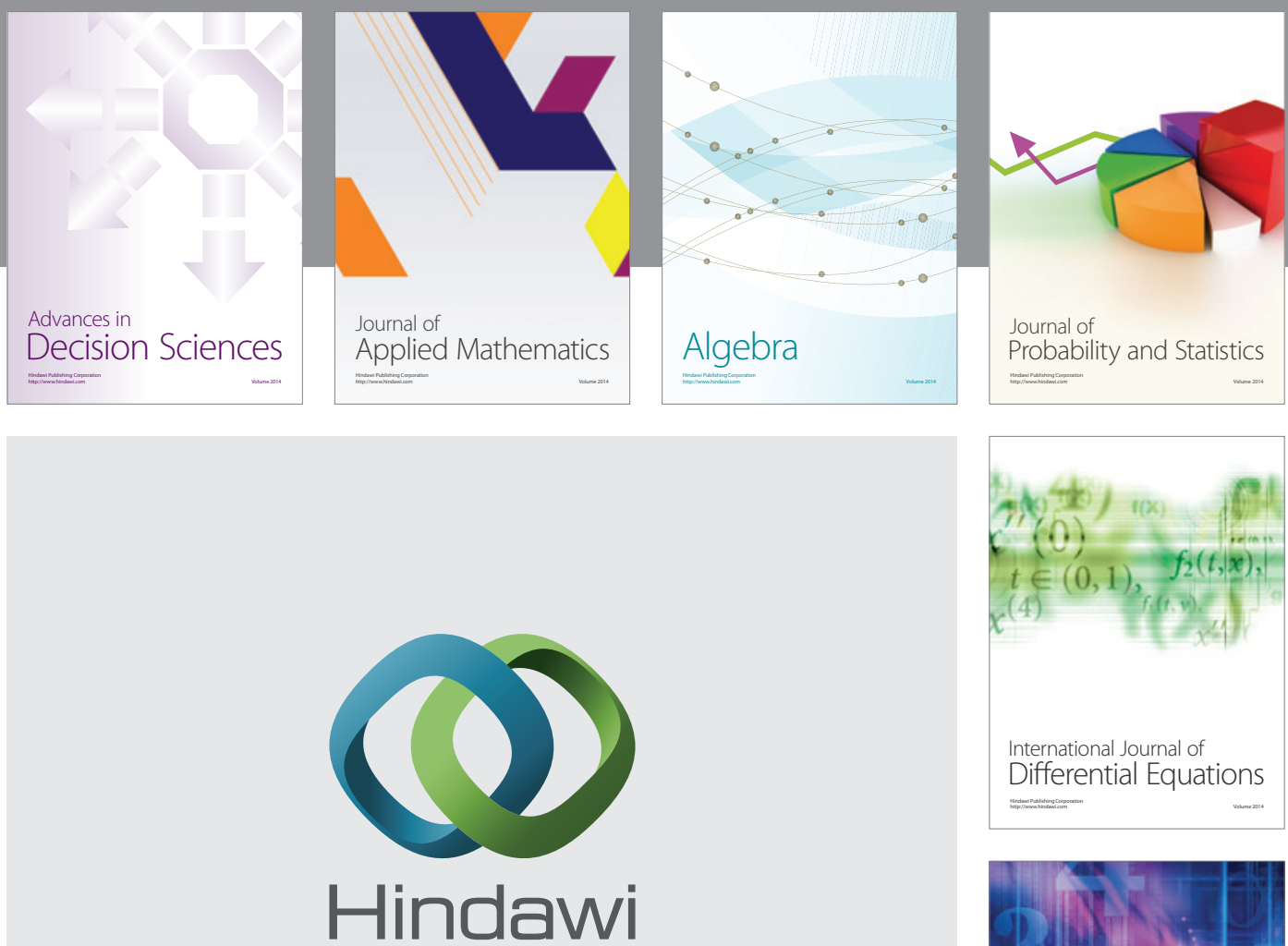

Submit your manuscripts at http://www.hindawi.com
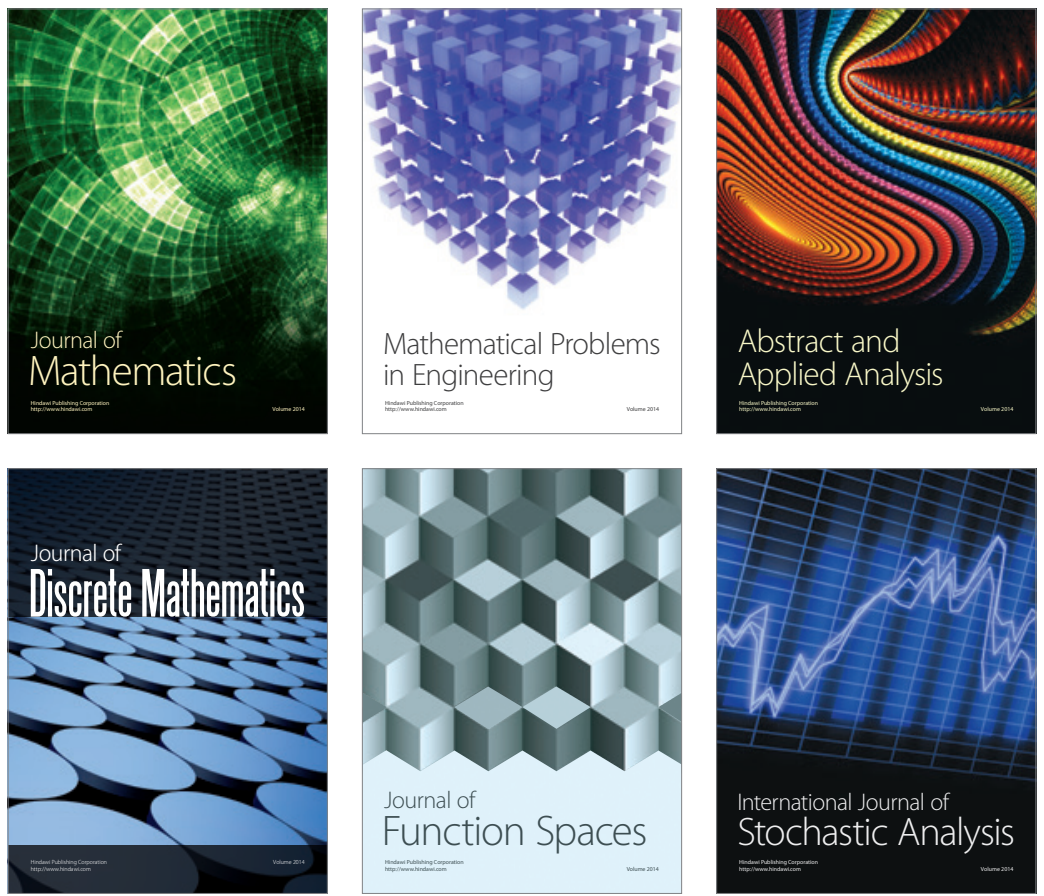

Journal of

Function Spaces

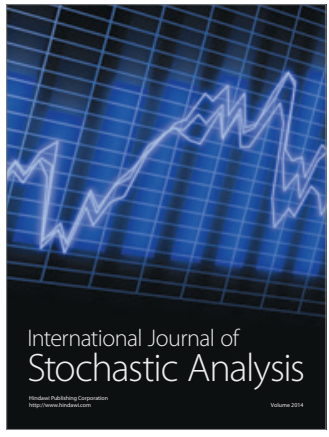

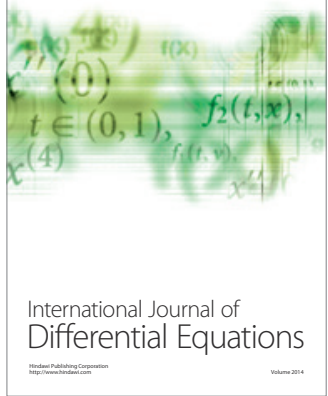
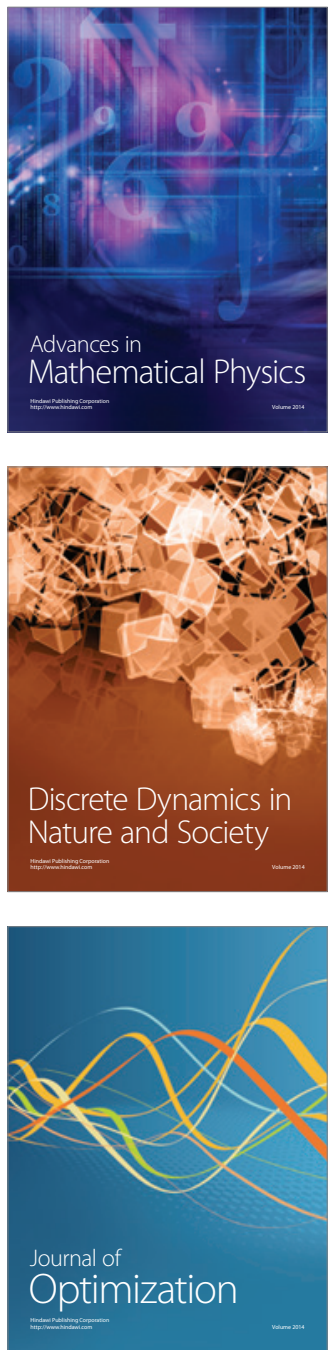